\section{Production of an intraocular device using 3D printing: an innovative technology for ophthalmology}

\author{
Primeiro dispositivo intraocular utilizando \\ impressão 3D: uma tecnologia inovadora \\ para oftalmologia
}

Three-dimensional (3D) printing, also termed additive manufacturing, allows the creation of 3D objects from digital files. In 3D printing, additive processes are used to lay down successive layers of material until the entire object is created. 3D printing technology has become widely used cost-effective alternative for small-scale productions and has numerous advantages; objects of almost any shape can be printed using extensive variety of different materials, including plastics, metals, ceramics, food, paper, wood filament, and biomaterials ${ }^{(1)}$

$3 \mathrm{D}$ technology is opening up new perspectives in research ${ }^{(2)}$ and has begun to revolutionize the field of Health Sciences by facilitating low-cost manufacturing and custom surgical devices, 3D models for use in preoperative planning and surgical education, and fabricated biomaterials. However, there is a lack of data regarding the viability of $3 \mathrm{D}$ printing for the production of surgical instruments ${ }^{(3)}$. Methods for the printing live cells, tissues, and organs, termed 3D bioprinting, is under development, with several studies demonstrating the utility of $3 \mathrm{D}$ bioprinting in the successful manufacturing of replicas of bones, ears, and blood vessels. The concept of 3D printing is relatively new in the field of ophthalmology ${ }^{(4,5)}$, with few publications evaluating its utility in this field ${ }^{(4)}$. Increased understanding of 3D technology will be of benefit to ophthalmologists.

The ability to produce a device using 3D printing technology for use in intraocular surgery remains unclear. The major issue faced by developers is biocompatibility, particularly in the case of producing intraocular devices. Studies involving 3D printing biocompatible materials are initially required to evaluate the feasibility of this approach. The United States Pharmacopeial Convention (USP) is a scientific, nonprofit organization that sets standards for the identity, strength, quality, and purity of medicines and biomaterials. Materials are ranked in six classes (I-VI) of biocompatibility. Biocompatible materials are assessed in terms of five categories related to medical approval, comprising cytotoxicity, genotoxicity, delayed-type hypersensitivity, irritation, and USP class VI, which includes tests for irritation, acute systemic toxicity, and implantation.

The second step in the validation of 3D printing in ophthalmology is the demonstration of precise device modeling at a resolution of approximately $600 \mu \mathrm{m}$, depending on what material is being printed. 3D models can be designed using either Maya, a comprehensive 3D animation software, or directly in the solid modeling computeraided design software, SolidWorks (Figure 1). Regardless of the 3D modeling software used, the models must be converted to an STL file to allow 3D printing. Stereolithography refers to the additive manufacturing technology used for producing model parts one layer at a time and represents a fast prototyping process for the creation of solid physical models directly from computer data. Prior to printing 3D models from STL files, designs must be examined for manifold errors, such as surfaces that do not connect or gaps in models. After all of these requirements have been met, 3D models can be printed. Additive processes typically reduce the time required for printing to just a few hours.

This process has allowed the development of Cana's Ring (CR), a 3D pupil expansion device; this was the first intraocular model

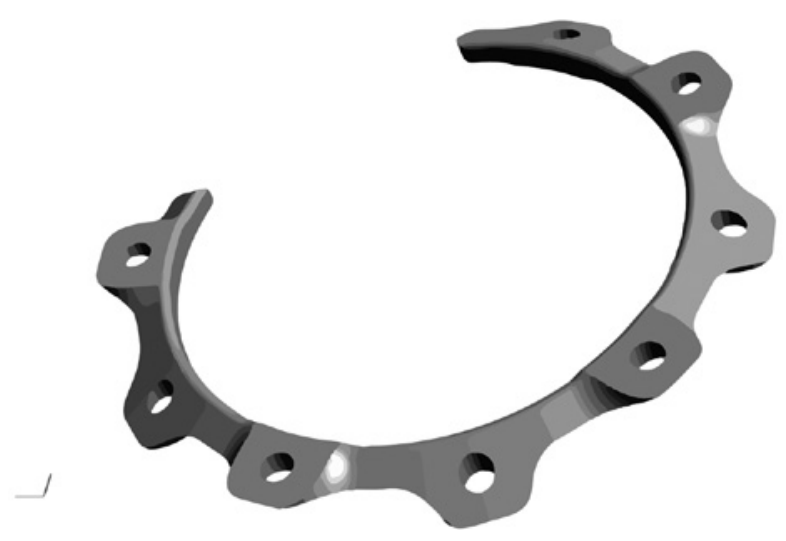

Figure 1. 3D model software image.

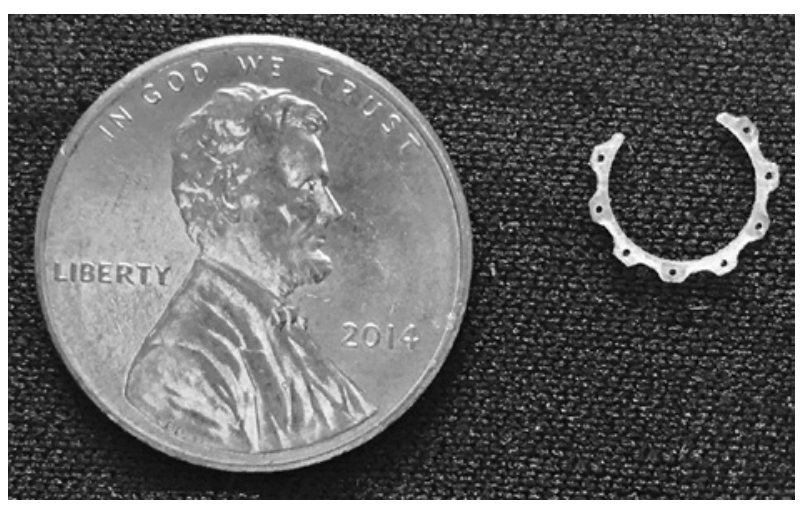

Figure 2. Cana's Ring 3D pupil expansion device.

designed and manufactured using 3D printing technology. The CR consists of a ring segment containing eight alternate parts (one side oriented up and one side oriented down) to embrace the pupillary edge (Figure 2). It is a 300 degree VisiJet ${ }^{\circledR}$ ring with a 60 degree region allowing the insertion of the ring into the eye and positioning along the same axis as the incision used during surgery. The device is inserted into the anterior chamber through a triplanar clear corneal incision. Immediately after the entire ring is situated within the anterior chamber, the ring is placed on the pupillary edge of the iris using the $\mathrm{CR}$ holes. This process is performed by inserting the down-oriented CR parts under the pupil edge and the up-oriented CR parts over the pupil edge. Pupillary dilatation to a diameter of $6.5 \mathrm{~mm}$ results when the entire ring is correctly placed along the pupillary edge of the iris. The use of the CR allows for the surgery for cataract to be performed using standard techniques.

Additive manufacturing represents a valid method of manufacturing ophthalmic models, devices, and instruments using biocompatible materials at a relatively low cost. This pioneering approach may open new perspectives for the use of 3D printing, an innovative technology with a substantial potential applicability in the field of ophthalmology. 
Submitted for publication: July 31, 2015

Accepted for publication: September 23, 2015

Glaucoma and Cataract Reference Center, Santa Casa de Misericórdia de Belo Horizonte, Belo Horizonte, MG, Brazil.

Department of Ophthalmology and Otorhinolaryngology, Universidade Federal de Minas Gerais, Belo Horizonte, MG, Brazil.

Department of Ophthalmology, Universidade Federal de São Paulo, São Paulo, SP, Brazil.

Funding: No specific financial support was available for this study.

Disclosure of potential conflicts of interest: Dr. Canabrava has a pending international patent application (PCT-WIPO) for the pupil-expansion device.

Corresponding author: Sérgio Canabrava. Santa Casa de Misericórdia de Belo Horizonte. Av. Brasil, 84, sala 1.303 - Belo Horizonte, MG - Brazil - E-mail: sergiocanabrava@hotmail.com

\section{REFERENCES}

1. Chia HN, Wu BM. Recent advances in 3D printing of biomaterials. J Biol Eng. 2015;

2. Jones N. Science in three dimensions: the print revolution. Nature. 2012:487(7405):22-3.

3. Rankin TM, Giovinco NA, Cucher DJ, Watts G, Hurwitz B, Armstrong DG. Three-dimensional printing surgical instruments: are we there yet? J Surg Res. 2014;189(2):193-7.

4. Schubert C, van Langeveld MC, Donoso LA. Innovations in 3D printing: a 3D overview from optics to organs. Br J Ophthalmol. 2014;98(2):159-61.

5. Huang W, Zhang X. 3D Printing: Print the future of ophthalmology. Invest Ophthalmol Vis Sci. 2014;55(8):5380-1.

\section{Publish your work on ABO}

\section{First Ophthalmology Journal With Free Full Content for Tablets. Check on App Store}

\section{Free Online Access}

www.scielo.br/abo

PubMed

JCR

SCOPUS 